\title{
A generic method for evaluating crowding in the emergency department
}

\author{
Andreas Halgreen Eiset ${ }^{1 *}$, Mogens Erlandsen², Anders Brøns Møllekær ${ }^{1}$, Julie Mackenhauer ${ }^{1}$ and Hans Kirkegaard ${ }^{1}$
}

\begin{abstract}
Background: Crowding in the emergency department (ED) has been studied intensively using complicated non-generic methods that may prove difficult to implement in a clinical setting. This study sought to develop a generic method to describe and analyse crowding from measurements readily available in the ED and to test the developed method empirically in a clinical setting.

Methods: We conceptualised a model with ED patient flow divided into separate queues identified by timestamps for predetermined events. With temporal resolution of 30 min, queue lengths were computed as $Q(t+1)=Q(t)+$ $A(t)-D(t)$, with $A(t)=$ number of arrivals, $D(t)=$ number of departures and $t=$ time interval. Maximum queue lengths for each shift of each day were found and risks of crowding computed. All tests were performed using non-parametric methods. The method was applied in the ED of Aarhus University Hospital, Denmark utilising an open cohort design with prospectively collected data from a one-year observation period.
\end{abstract}

Results: By employing the timestamps already assigned to the patients while in the ED, a generic queuing model can be computed from which crowding can be described and analysed in detail. Depending on availability of data, the model can be extended to include several queues increasing the level of information. When applying the method empirically, 41,693 patients were included. The studied ED had a high risk of bed occupancy rising above $100 \%$ during day and evening shift, especially on weekdays. Further, a 'carry over' effect was shown between shifts and days.

Conclusions: The presented method offers an easy and generic way to get detailed insight into the dynamics of crowding in an ED.

Keywords: Crowding, Emergency department, ED, Generic, Method, Model, Queue, Patient flow

\section{Background}

Crowding is a major health concern and an increasing problem for Emergency Departments (EDs) internationally [1-3]. Crowding is defined as a situation where treatment demands exceed available resources and is associated with increased mortality, increased treatment costs and over-all reduced quality of care [4-8].

Crowding can be conceptualised as a model of patient flow, which incorporates factors that influence on or are affected by crowding [9]. Any such model can be classified on a continuum from high generalisability with abstract information outputs (i.e. "level 1") to highly specific with low generalisability (i.e. "level 4") [10].
When developing a new model, researchers should acknowledge at which level they want their model to operate; balancing the need for application in various settings (level 1) against the need for a model drawing very specific conclusions (level 4). A simple generic method for evaluation of crowding that allows easy implementation into various EDs is in high demand [11-13].

The aim of this study was to develop a method that meets the criteria for an ideal universal measure for crowding being generalisable in measurement, definition and validity as described by Dr. Pines [14]. The proposed method was tested empirically in a clinical setting.

* Correspondence: ahe@clin.au.dk

${ }^{1}$ Research Centre for Emergency Medicine, Aarhus University Hospital,

Aarhus, Denmark

Full list of author information is available at the end of the article

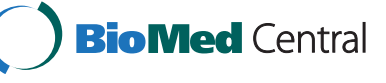

(c) 2016 The Author(s). Open Access This article is distributed under the terms of the Creative Commons Attribution 4.0 International License (http://creativecommons.org/licenses/by/4.0/), which permits unrestricted use, distribution, and reproduction in any medium, provided you give appropriate credit to the original author(s) and the source, provide a link to the Creative Commons license, and indicate if changes were made. The Creative Commons Public Domain Dedication waiver (http://creativecommons.org/publicdomain/zero/1.0/) applies to the data made available in this article, unless otherwise stated. 


\section{Methods}

\section{Study design and population}

An emergency department (ED) has been defined as a hospital department accessible to patients with medical emergencies staffed by multidisciplinary clinical personnel ready to provide immediate stabilisation and care [15]. The processes that a patient must undergo from the time of arrival to departure characterise patient flow in the ED [9]. We defined a patient as being present in the ED from the time of electronic registration at the front desk (arrival) to the time of electronic registration of departure from the ED unit.

Since this study aimed at developing a generic method using readily available data to describe crowding we started by setting up a model of the ED depending only on the two most reliable timestamps: time of arrival and time of departure. This "black box" model has one queue representing ED census at a given time (Fig. 1a).

From the patient flow data, we created an aggregate data set by dividing the study period into intervals of 30 min and counting the number of arrivals $A(t)$ and departures $\mathrm{D}(\mathrm{t})$ in each interval $\mathrm{t}$ (Table 1). From the number of arrivals and departures in each interval, the queue length at the beginning of the next interval could be calculated as: $\mathrm{Q}(\mathrm{t}+1)=\mathrm{Q}(\mathrm{t})+\mathrm{A}(\mathrm{t})-\mathrm{D}(\mathrm{t})$, using $\mathrm{Q}(0)=0$ (the initial queue length).

For every shift (day, evening and night) of each day in the study period the maximum queue (max queue) length was found. Days were defined as beginning by the day shift at 7 a.m. with every shift lasting eight hours, thus reflecting the clinical setting. To allow for steady state in the queuing system, all analysis of queues were made discarding the first 24-h, i.e. the first three shifts, of the study period.

To explore possible extensions of the model, thus increasing the level of information on throughput processes, we conceptualised an idealised patient flow by dividing the stay in the ED into four queues based on clinical relevance and structural challenges: A queue between arrival and start of initial assessment by an EDnurse (Q1), a queue before evaluation by a doctor (Q2), a queue before completion of examination and treatment in the ED (Q3) and finally a queue arising for patients who are waiting to leave the ED (Q4). With few exceptions, e.g. trauma call patients, every patient presenting in the ED would go through these steps in that exact order. This is in line with the conceptual model proposed by Asplin and colleagues for ED throughput [9]. Since it was not possible to get verbatim timestamps for some of these factors (i.e. start of initial assessment, first evaluation by a doctor and completion of treatment), a number of surrogate-markers were decided upon: time of first triage, first re-assessment of vital signs "TOKS" value (a Danish early warning system based on systematic assessment of vital signs [16]) and last TOKS value. Figure 1b offers a graphical representation of this model.

In applying the method we used an open cohort design with prospectively collected data on every patient presenting to the ED from January $1^{\text {st }}$ to December $31^{\text {st }}$ 2013. The number of arrivals during the study period determined the sample size and patients were followed from ED admission to ED discharge as registered in the Electronic Health Records (EHR). EHR contains patient administrative data (e.g. age, gender, time of admission and discharge) and clinical patient data (e.g. triage score and diagnosis code). Utilising the "black-box" model for patient flow we computed the aggregate data set $(N=$ $17,520)$ and max queue length for each shift (3) of each day $(365$, i.e. $3 * 365$ values of max queues). Likewise, queue lengths for each of the four queues in the extended model of idealised patient flow in the ED were calculated for each day and shift (i.e. $4 * 3 * 365$ values of max queues).

\section{Study site}

We applied the method to the ED at Aarhus University Hospital, Denmark. The hospital had an uptake area of

a

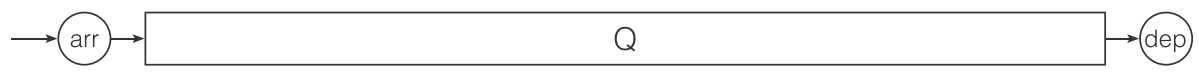

b

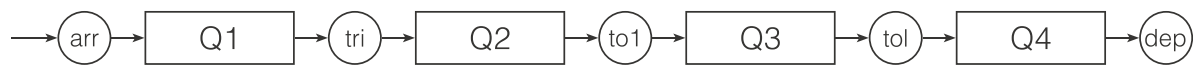

Fig. 1 Conceptual models for the patient flow through the ED. a A simplified "black box" model of the patient flow to and from the ED depending only on time of arrival and departure. b By utilising surrogate-markers queues representing patients waiting on start of examination (Q1, waiting on triage), evaluation by a doctor (Q2, waiting on first TOKS), completion of examination/treatment in the ED (Q3, waiting on last TOKS) and leaving the ED (Q4) were proposed. All but a few patients in the ED would follow this flow in that exact order. Abbreviations: arr, arrivals; tri, triage; to1 first TOKS; tol, last TOKS; dep, departure, Q, queue 
Table 1 Variables obtained for each patient in each 30-min interval

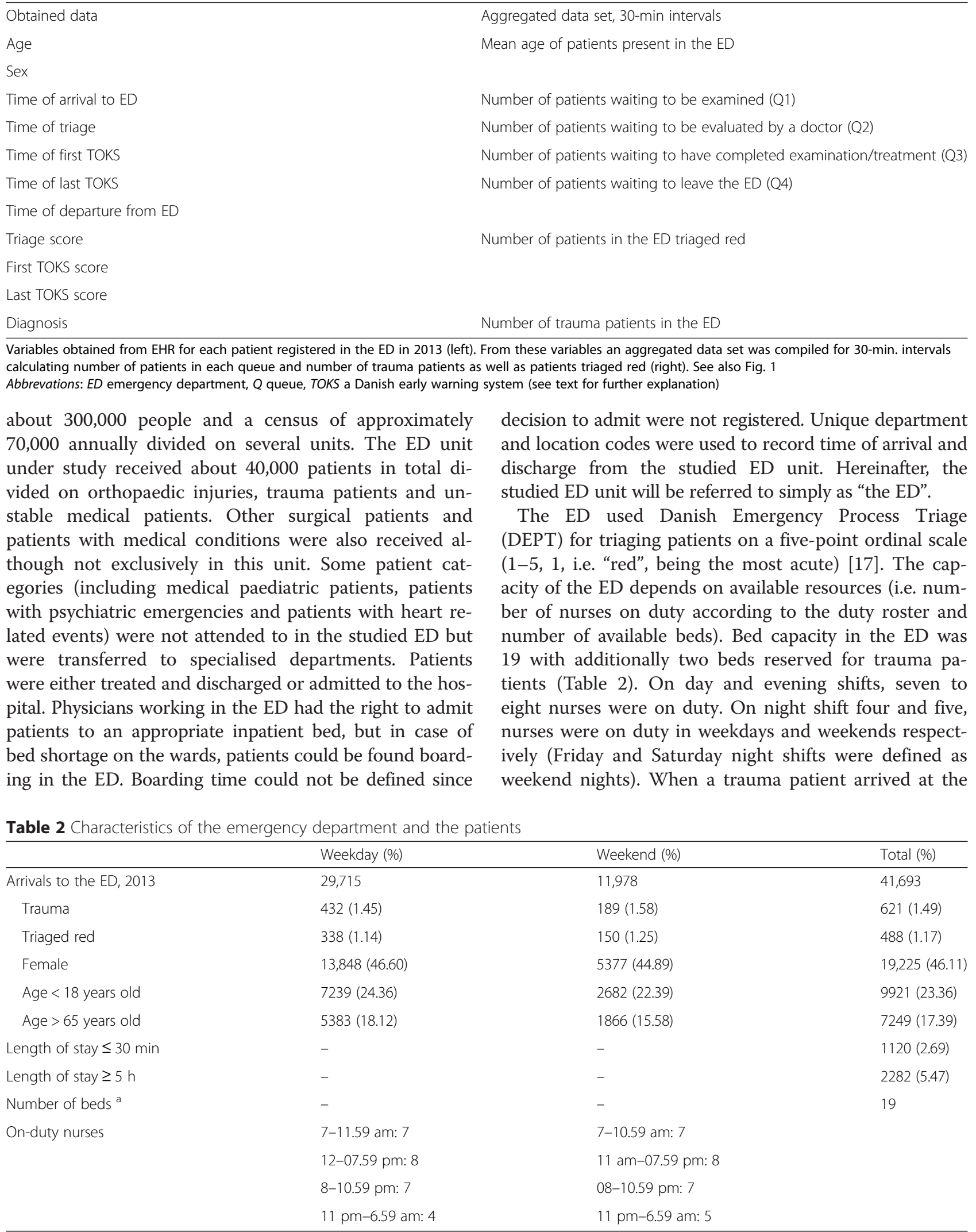


ED, one to three nurses would be allocated to this patient thus lowering the nurse-capacity in the remaining ED. At times of special need one nurse could be transferred to the ED to increase the nurse-capacity though no formal staffing policy for this was in place.

\section{Statistical methods}

Due to highly skewed distributions, we used nonparametric statistical methods to evaluate queue lengths: Kruskal-Wallis rank sum test to compare the distributions of the maximum daily queue length in each of the three shifts and between weekday and weekends and Spearman's rank sum correlation test (Spearman's rho) to evaluate correlations between maximum queue lengths in successive shifts. We applied Fisher's index of dispersion to evaluate Poisson distribution of the data. Data management and statistics were done using $R$, version 3.1.2 ( $\mathrm{R}$ Foundation for Statistical Computing, Vienna, Austria).

\section{Results}

From the timestamps of arrivals and departures a simple method could be set up and risk of crowding and its predictors computed. It was possible to extend the model by separating the patient flow in the ED into clinically meaningful queues allowing for increased level of information. All codes necessary for the data management and analyses presented including an example data set are freely available (see section Availability of data and materials).

We tested the proposed method empirically on the "black box" model: A total of 41,693 arrivals were registered in the ED during the study period (Table 2). About $70 \%$ of all arrivals happened on weekdays. Of all visits, $2.7 \%(1120 / 41,693)$ spend $30 \mathrm{~min}$ or less in the ED and $5.5 \%(2282 / 41,693)$ spend more than $5 \mathrm{~h}$ in the ED. Figure 2 offers detailed insights into the queue dynamics, illustrated by a queue of 42 patients, which arose on November 18th 2013. On this day the longest queue during the study period was observed, but any days of interest could have been chosen. The figure permits direct temporal observation of the queue length and its relation to the number of arrivals and departures indicating whether the queue length grows due to external or internal processes. If a fall in departures precedes prolongation of the queue length we expect internal processes (i.e. throughput) slowing down to be the main driver of the queue length. Conversely, a steep rise in arrivals (i.e. input) preceding a prolonged queue indicates external processes to be the driver of the queue length. It is always interplay between input, throughput and output, but in the example given in Fig. 2 external processes - i.e. the number of arrivals - appears to be the main driver of the queue length. The distributions of the number of arrivals and the queue lengths are shown in Fig. 3. These distributions could not be described by Poisson

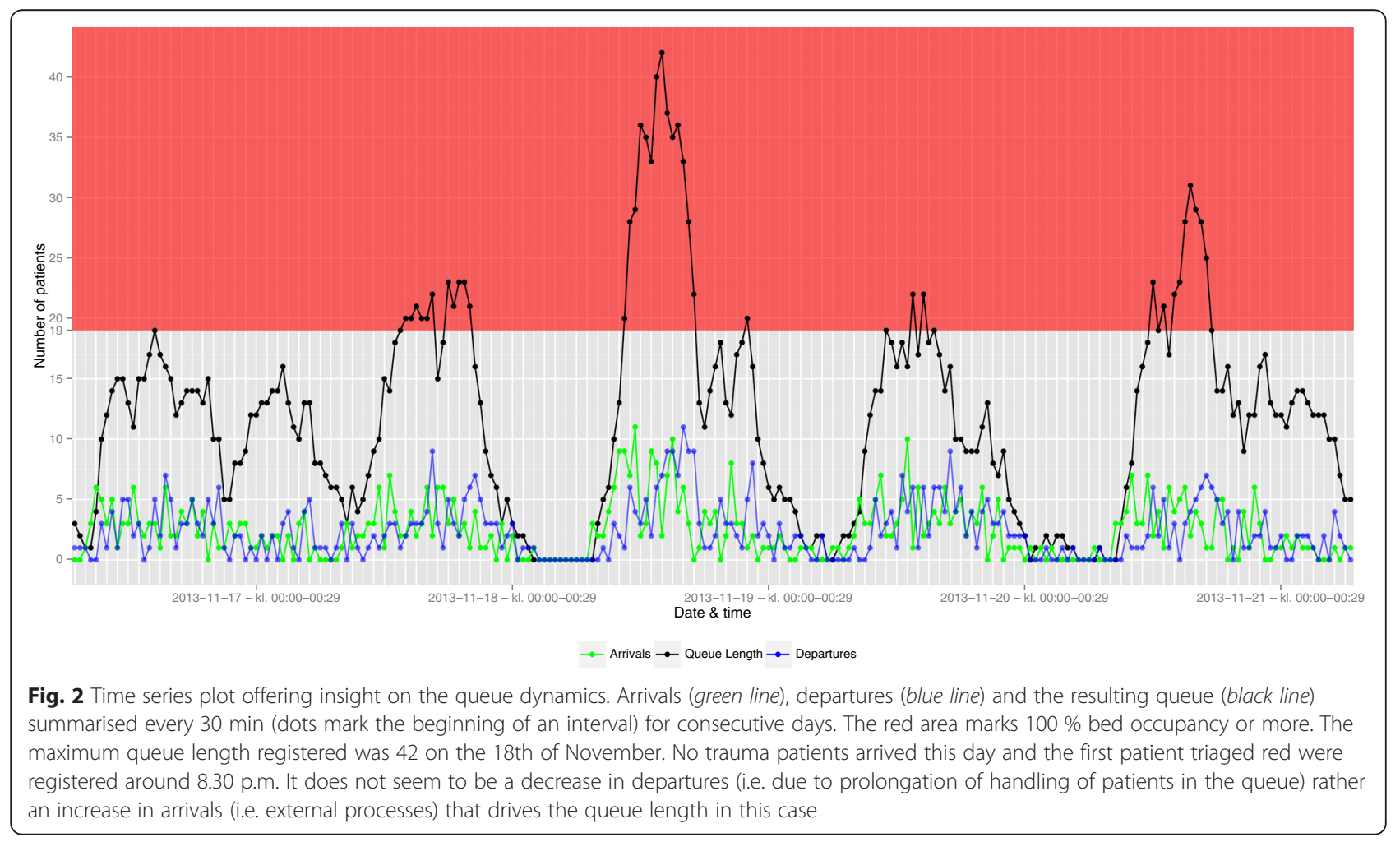



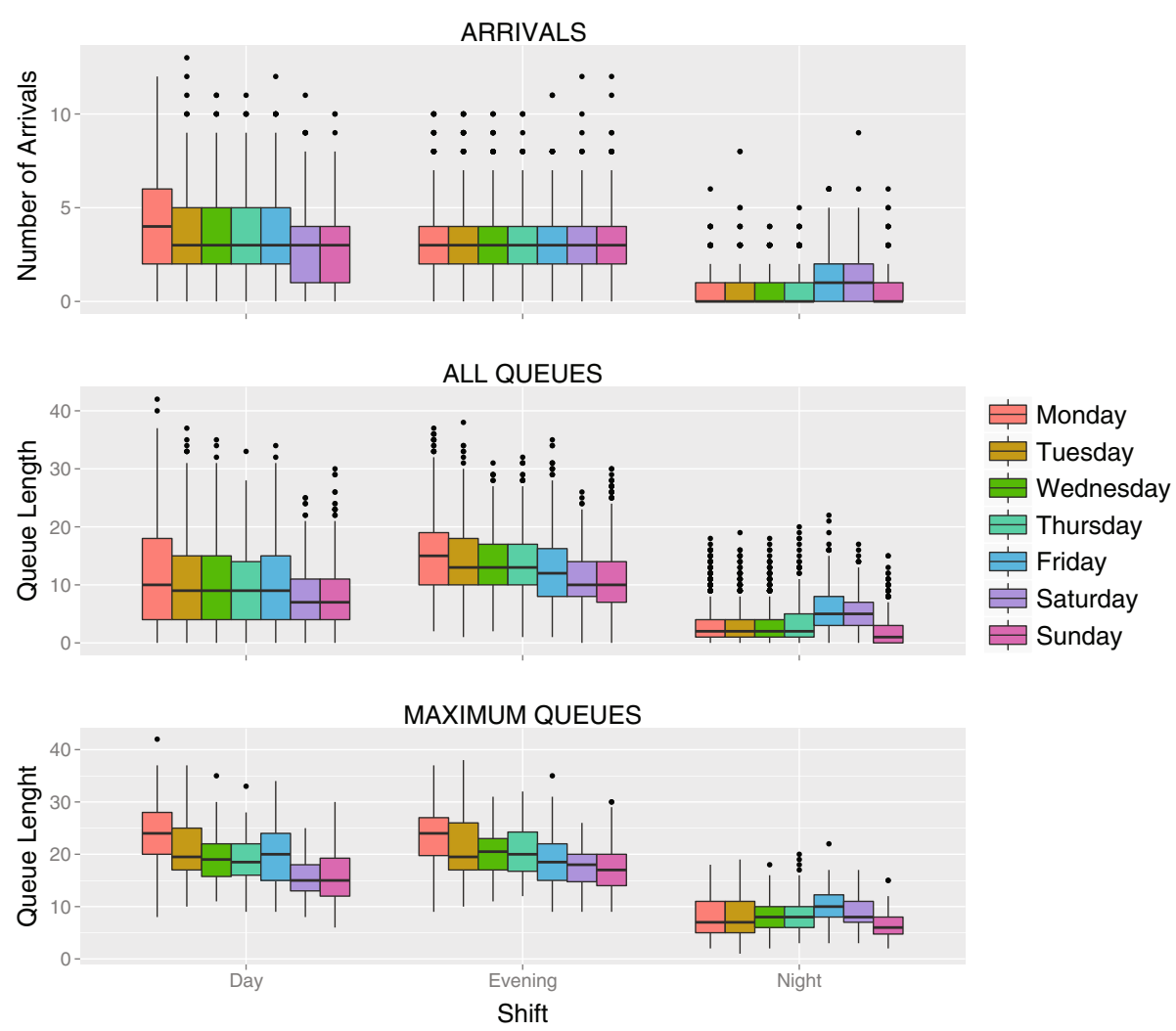

Fig. 3 Boxplot of number of arrivals, queue lengths and maximum queue lengths per day in 2013. The distribution of arrivals and queue length were non-normal with over-dispersion. Queue lengths ("all" as well as "maximum") and arrivals differed between shifts (Kruskal-Wallis: $p<0.0001$ ). This was true as well between weekdays and weekends for maximum queues and arrivals, but for all queues an association was found (Kruskal-Wallis: $p=0.1309$ )

distributions since Fisher's index of dispersion consistently showed over-dispersion, indicating negative binomial distributions would be a better fit. The distribution of max queues on weekdays vs. weekends and the three shifts differed significantly with Kruskall-Wallis: $p<$ 0.0001 for both. On weekdays in day and evening shift the risk that the queue would grow to and above the maximum bed capacity at least once during the shift was more than 50 \% (Fig. 4). On weekends this risk dropped to 21 and $27 \%$ respectively. In night shifts the risk plummeted to around $1 \%$ disregarding weekday/weekend. Seasonality (summer: April - September) was observed (Kruskall-Wallis: $p=0.0233$ ), but only conveyed minor changes in risk of reaching a $100 \%$ bed capacity when stratified for (e.g. the risk on weekday day shift were $54 \%$ overall and $58 \%$ and $50 \%$ summer and winter respectively). The absolute number of times with crowding can be inferred from the percentages given in Fig. 4. Likewise, this frequency can be visualised with modifications to Fig. 2.

When restricting the analysis to include the observed max queue for each day and each shift, it was found that the median queue length was 20 patients with an interquartile range (IQR) of 8.00 patients on weekdays day and evening shifts, and $7(\mathrm{IQR}=5.00)$ patients in the night (Table 3). On weekends the shift medians were 15 $(\mathrm{IQR}=6.00), 18(\mathrm{IQR}=6.00)$ and $9(\mathrm{IQR}=4.25)$ patients respectively. This gives a median patient-to-nurse ratio of 2.9 in day shifts on weekdays as the maximum and 1.8 on night shifts as the minimum ratio detected.

Max queue lengths in day shifts were found to correlate positively with $\max$ queue lengths the following evening shifts $(\rho=0.67, p<0.0001)$ (Fig. 5). This relationship - a 'carry over' effect of queue length between shifts - also applied to evening and night shifts $(\rho=0.20$, $p<0.0001$ ), however, it did not apply to night and the following day shifts $(\rho=-0.02, p=0.65)$. Max queue lengths of one day correlated positively with max queue lengths the following day $(\rho=0.24, p<0.0001)$.

Unfortunately, in the explorative phase of the project it became clear that triage and TOKS data could not be retrieved for a large proportion of patients. For instance, all but arrival and departure registrations were missing in $71 \%$ of the patients (Fig. 6). Thus, the abovepresented calculations could not be repeated for the extended model. In an ED with data that supports this 


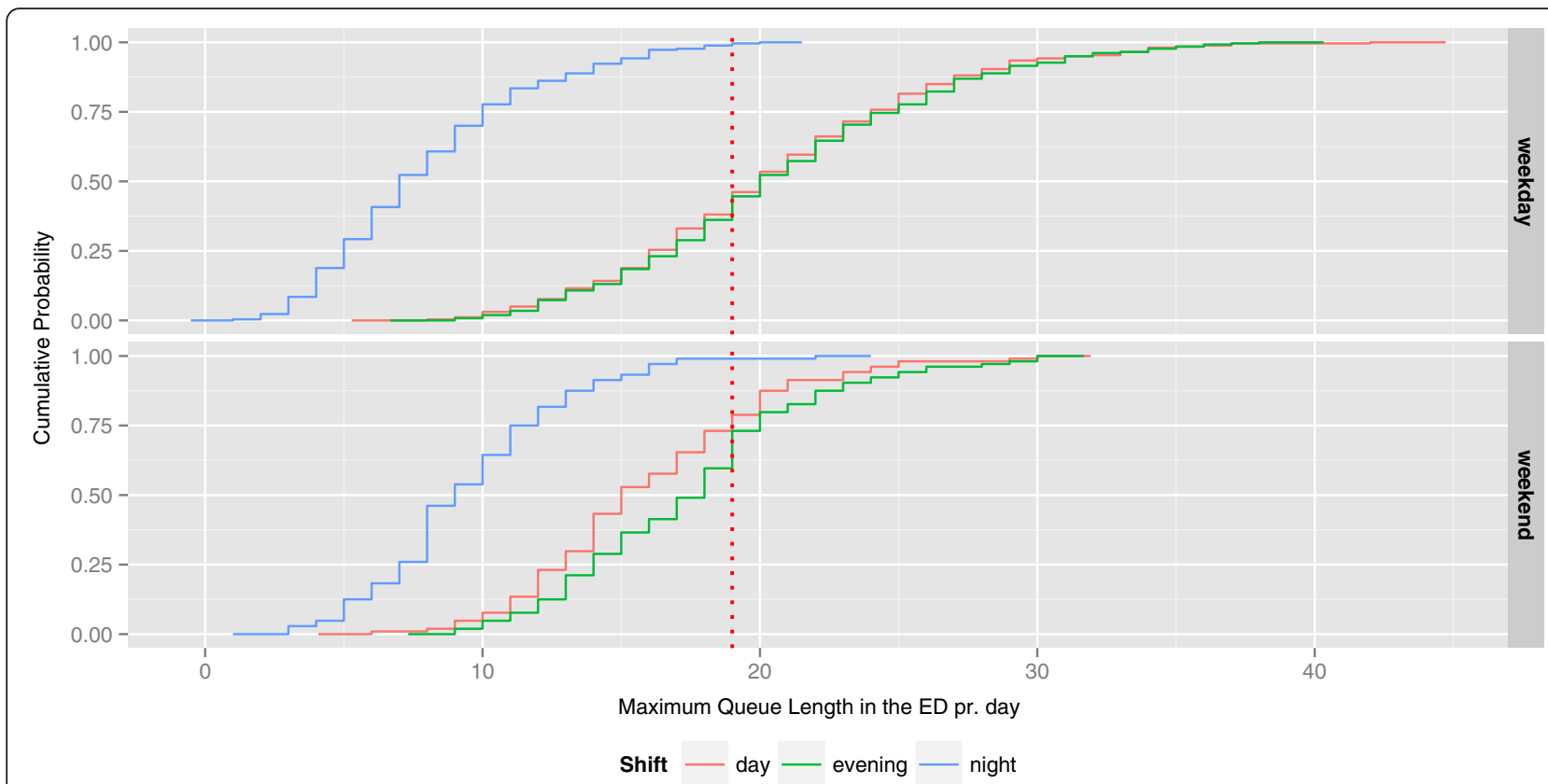

Fig. 4 The empirical cumulative distribution function of max queues in the ED. The dotted red line indicates the point of $100 \%$ bed occupancy (i.e. a queue length of 19). On weekdays there was a risk that 19 or more patients being present in the ED of $54 \%, 56 \%$ and $<1 \%$ at some point during day, evening and night shift respectively. In weekends the risk was $21 \%, 27 \%$ and $<1 \%$

model each of the four queues could be analysed in a similar manner.

\section{Discussion}

The main purpose of our study was to develop a generic method to describe and estimate crowding in an ED. By utilising only time of arrival and time of departure it was possible to get detailed insight into the dynamics of the queue and the resulting crowding in the ED. Since the method only relies on timestamps and patient census, it is the question at hand and data availability that sets the limit to the practical implementation of the method: A finer temporal resolution can be chosen, other time stamps can be used to define the queue of interest and several queues be combined to elaborate on the queues during patient flow (e.g. Fig. 1b). Likewise, the $\max$ queue can be related to one or more groups of staff in the ED and/or to patients of interest. We encourage fellow researchers to build on and modify the programming code for the method to suit the research question at hand.

The max queues represent the 30-min time period of each shift on each day with most patients in the ED. The interpretation is exemplified by the empirical cumulative distribution function (Fig. 4): It expresses the risk of at least one interval with a given queue length. Similarly, the positive correlation of max queues between shifts following each other means that, if the maximum queue during a shift was long, it was likely that a long queue would arise at least once during the following shift. When analysing crowding we are interested in what happens at times with prolonged queues. The observations

Table 3 Characteristics of maximum queue lengths per day in 2013

\begin{tabular}{lllllll}
\hline & & $\%$ of time with $\geq 19$ patients & Min & Max & Median (IQR) & Median patient/nurse ratio \\
\hline Day shift (7 am-02.59 pm) & Weekday & $54 \%$ & 8 & 42 & 20 (8.00) & 2.9 (2.5 when 8 nurses) \\
& Weekend & $21 \%$ & 6 & 30 & $15(6.00)$ & 2.1 (1.9 when 8 nurses) \\
Evening shift (3 pm-10.59 pm) & Weekday & $56 \%$ & 9 & 38 & $20(8.00)$ & $2.5(2.9$ when 7 nurses) \\
& Weekend & $27 \%$ & 9 & 30 & $18(6.00)$ & $2.1(2.4$ when 7 nurses) \\
\multirow{2}{*}{ Night shift (11 pm-6.59 am) } & Weekday & $<1 \%$ & 1 & 20 & $7(5.00)$ & 1.8 \\
& Weekend & $<1 \%$ & 3 & 22 & $9(4.25)$ & 1.8 \\
\hline
\end{tabular}

Characteristics of max queues and the median patient/nurse ratio computed from this. When observations were restricted to the time of each day and shift with the highest patient load there were considerable differences in the patient pr. nurse ratio. Friday and Saturday night were considered part of the weekend Abbreviation: IQR interquartile range 


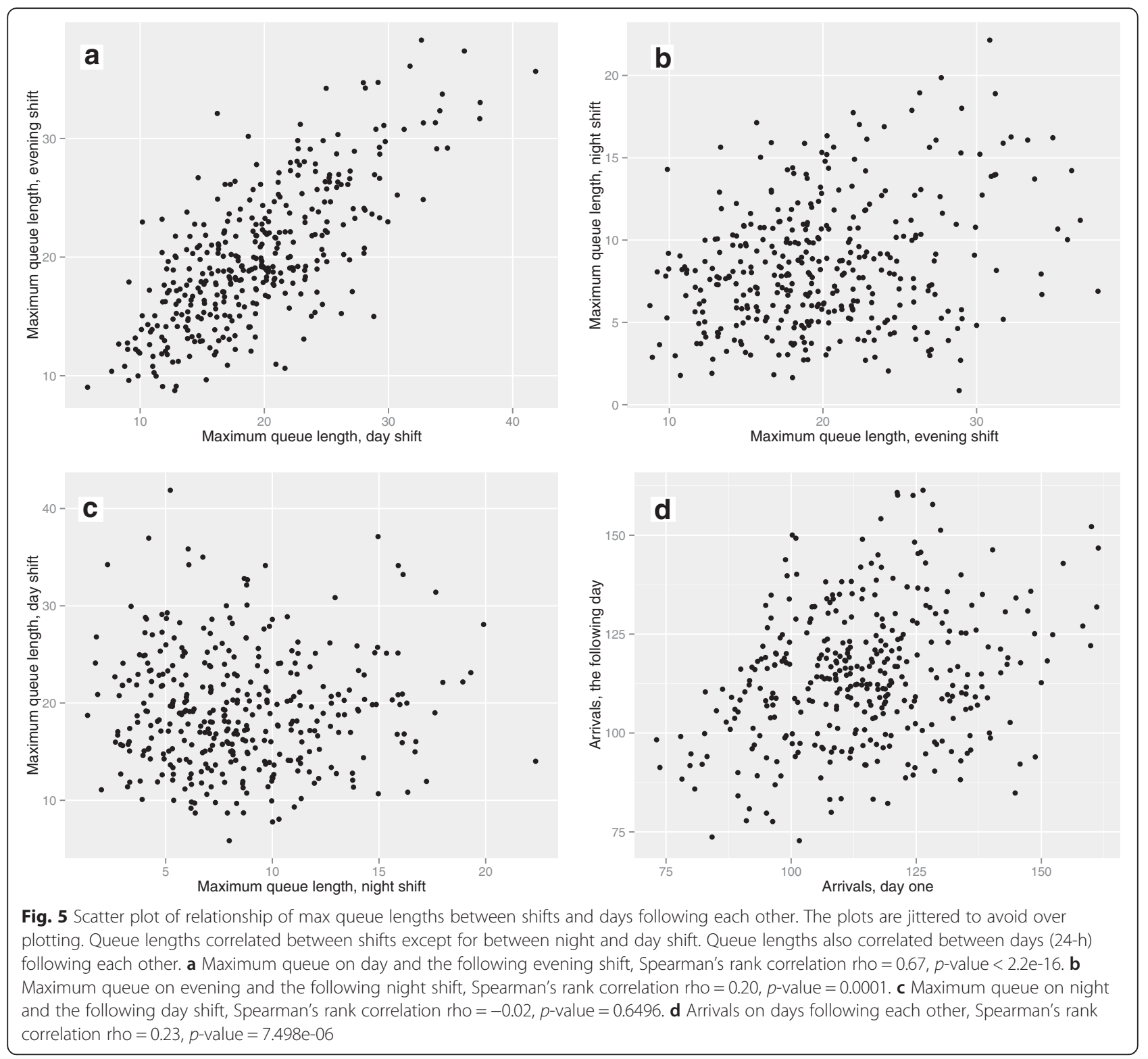

with minor queues can be thought of as noise to the signal of interest. On the other hand if only observations with queue lengths above a certain threshold were chosen, variation would be lost and with it our ability to make inferences. To the knowledge of the authors, no other research has been done on maximum queues in the ED.

Crowding has been measured in a number of ways ranging from subjective assessment by ED staff [18] to objective measurements [19-24] and combinations of the two $[12,15]$. To add to this, the threshold defining when crowding occurs is absolute in some studies [15, 19-21] using objective measurements for crowding and relative in others [24]. When crowding is defined subjectively the external generalisability should be questioned: What is perceived as crowding in one ED might be considered otherwise in another [25-27]. Objective definitions however, can be criticised for oversimplifying a complex interplay between numerous factors [9]. When the threshold for crowding is relative it is inevitable that crowding will occur a set percentage of the time, disregarding that the ED might never reach full bed capacity, experience the adverse effects of crowding etc. This limits possible research questions to concern effects of "above-normal" number of patients in the ED. Finally, dynamic measurement of crowding (i.e. several measurements during a patient's stay in the ED) is preferable to a static measurement (i.e. a single measurement e.g. when arriving at the ED) [24]. Methods requiring highly detailed administrative data and/or specialised 


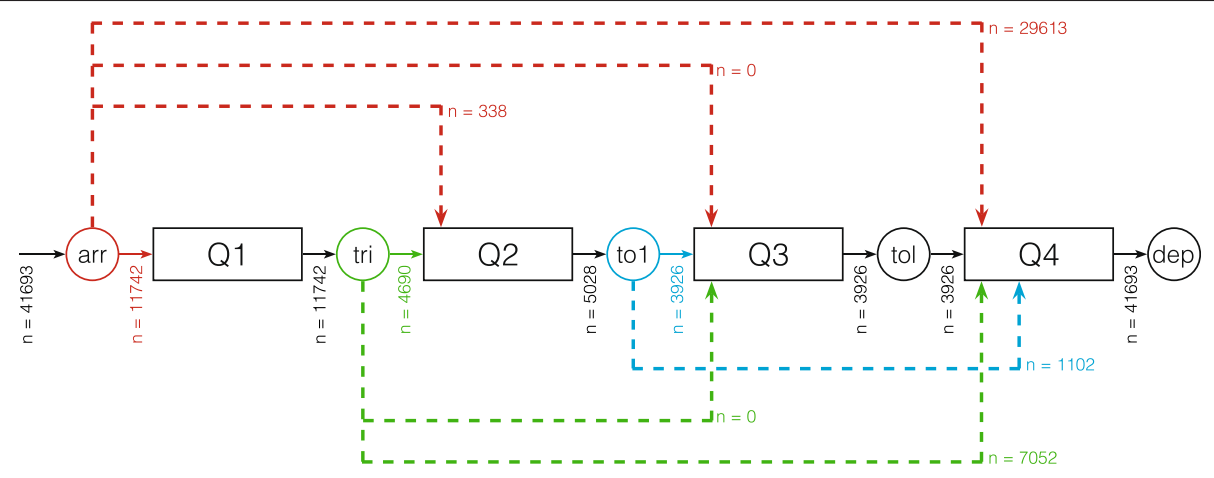

Fig. 6 Examination of the empirical patient flow. Arrows indicates patient flow going from a timestamp to a queue. 41,693 patients arrived at the ED. Of these $11,742(28.2 \%)$ patients got a triage score registered, $338(0.8 \%)$ patients did not get a triage score but did get a first TOKS score, and 29,613 (71.0 \%) patients had neither triage nor TOKS values registered. Of the 11,742 patients that did get a triage score 4690 also got a first TOKS score. Thus, a total of 5028 (12.1\%) patients had a registered value for first TOKS. Only 3926 (9.4\%) patients had a registered value for all the factors in the idealised model for the patient flow. It was therefore decided to do the further analysis on the "black box" model with only arrival and departure as factors, until better completeness of the data could be obtained. Abbreviations: n, number of patients; arr, arrivals; tri, triage; to1, first TOKS; tol, last TOKS; dep, departure; Q, queue

insight into statistical programming are seldom implemented in a clinical setting, are found to have low external validity and are not found to be superior to ED occupancy level in describing crowding [11, 13, 21, 28-30]. Likewise, if a method relies on specific assumptions about data, researchers must ensure that such assumptions are met before implementing the method. Failing to do so could result in deceptive conclusions. As an example, we found that arrivals did not follow a Poisson distribution, but rather a negative binominal distribution. This is in opposition to what is assumed in many studies on ED crowding $[23,24,31]$ and could render such methods errorprone in our setting. Therefore, we sought to develop a novel method for quantifying crowding in an ED from simple and readily available parameters with occupancy level as a central measurement of crowding and a temporal resolution of 30-min.

One example of direct actions based on our proposed method is the ability to anticipate crowding: If the max queue in a day shift is long the 'carry over' effect mean that a long queue are likely to arise during the following evening shift. This will allow interventions to counter crowding before it arises. Such prediction tool could be implemented and evaluated using the Plan-Do-Study-Act tool [32].

Some limitations must be addressed. Arrival and departure time were recorded for every patient in the study and are generally considered very accurate, and possible errors in these timestamps were most likely random. If they were systematic, e.g. if at busy times the arrivals were systematically collected and registered with delay all at the same time, it could seriously bias the findings towards longer queues. Other indicators of flow were considered, amongst others time of diagnosis, time of first medicine prescription, time of ordering of $\mathrm{x}$-ray and time of ordering blood tests. All of these were found to be particularly liable to "measurement" bias in that they would very often, and depending on the individual doctor, be registered later than actually effectuated, and were thus rejected. As with any standardised analysis model, this is an approximation of real life allowing a detailed overview of the system. Although very few assumptions are necessary with this method, complex organisational and psychological interactions between the factors are likely to play important roles in an ED; not least in times with crowding [33]. Such interactions have not been taken into account.

For the results of applying the method, it must be noted that EHR is a secondary data source primarily for clinical use. Since the data collection is not under the control of the researchers the quality of EHR data for research can be questioned [34]. On the other hand, the fact that data were collected as part of the clinical work without this research project in mind could be argued to lower the risk of introducing selection and/or information bias and thus strengthen the validity of the results. Further, the number of nurses on duty was derived from the duty roster and not the actual number of nurses meeting in each shift of each day. These numbers could differ e.g. due to illness. With a study period of one year we found it not to be feasible to allow for such variations but if investigating shorter periods of time this could be an option to strengthen the internal validity of the results. Finally, only the nurse capacity was included in the presented results leaving other valuable resources in the ED - such as doctors - unaccounted for. We encourage future research to focus on the possibility to extend the method to include several sub-queues (e.g. as proposed in Fig. 1b) and to explore the method in a multicentre trial.

In summary, building on the recommendations for dynamic measurement of crowding put forth by McCarthy 
et al. [24] we present a highly generic method relating ED capacity (beds and nurses) to dynamically measured ED census, setting no threshold for crowding. Our model is highly generalisable and easy to implement in diverse settings.

\section{Conclusion}

We put forward a generic method for evaluating crowding in an ED from readily available data. This allows for detailed analysis of crowding: its impact and associations in any specified ED. It offers insight on the dynamics of crowding and allows for further investigation of predictors of crowding.

\section{Abbreviations}

ED, emergency department; EHR, Electronic Health Records; IQR, interquartile range

\section{Acknowledgements}

The authors thank Jonas Rosendal Bager-Elsborg for his help in retrieving the data, and Malene Østerby Skov for her assistance in applying the method to the ED.

\section{Funding}

Aarhus Universitets Forskningsfond (DK); Fonden af 1870; Familien Hede Nielsens Fond. The funding bodies played no role in the design of the study, the collection, analysis, interpretation of data, or in writing the manuscript.

\section{Availability of data and materials}

Under Danish law raw data obtained from EHR cannot be published. The R-code for the data management as well as the analysis is available together with a constructed example of the dataset in the GitHub repository, https:// github.com/eiset/Crowding_code.git

\section{Authors' contributions}

All authors participated in conceiving and design of the study and helped to draft the manuscript. AHE coordinated the project, performed all data management and analysis and drafted the manuscript. ME set up the analysis plan. All authors read and approved the final manuscript.

\section{Competing interests}

The authors declare that they have no competing interests.

\section{Consent for publication}

Not applicable

\section{Ethics approval and consent to participate}

In accordance with Danish law permission to conduct the study has been granted from the Danish Data Protection Agency (2007-58-0010) and the Danish Health and Medical Authorities (3-3013-552/1/). Register based studies requires no further approval in Denmark. We report in adherence with the Strengthening the Reporting of Observational Studies in Epidemiology statement [35].

\section{Author details \\ ${ }^{1}$ Research Centre for Emergency Medicine, Aarhus University Hospital, Aarhus, Denmark. ²Department of Public Health, Section of Biostatistics, Aarhus University, Aarhus, Denmark.}

Received: 14 July 2015 Accepted: 22 May 2016

Published online: 14 June 2016

\section{References}

1. Derlet RW. Overcrowding in U.S. EDS: a critical condition. Cal J Emerg Med. 2002;3(2):24-6.

2. Pines JM, Pilgrim RL, Schneider SM, Siegel B, Viccellio P. Practical implications of implementing emergency department crowding interventions: Summary of a moderated panel. Acad Emerg Med. 2011; 18(12):1278-82. doi:10.1111/j.1553-2712.2011.01227.x.

3. Schiff GD. System dynamics and dysfunctionalities: levers for overcoming emergency department overcrowding. Acad Emerg Med. 2011;18(12):1255-61. doi:10.1111/j.1553-2712.2011.01225.x.

4. Fee C, Hall K, Morrison JB, Stephens R, Cosby K, Fairbanks RT, et al. Consensus-based recommendations for research priorities related to interventions to safeguard patient safety in the crowded emergency department. Acad Emerg Med. 2011;18(12):1283-8. doi:10.1111/j.1553-2712. 2011.01234.x.

5. Schuur JD, Hsia RY, Burstin H, Schull MJ, Pines JM. Quality measurement in the emergency department: past and future. Health Aff (Millwood). 2013; 32(12):2129-38. doi:10.1377/hlthaff.2013.0730.

6. Bernstein SL, Aronsky D, Duseja R, Epstein S, Handel D, Hwang U, et al. The effect of emergency department crowding on clinically oriented outcomes. Acad Emerg Med. 2009;16(1):1-10. doi:10.1111/j.1553-2712.2008.00295.x.

7. Carter EJ, Pouch SM, Larson EL. The relationship between emergency department crowding and patient outcomes: a systematic review. J Nurs Scholarsh. 2014;46(2):106-15. doi:10.1111/jnu.12055.

8. Moskop JC, Sklar DP, Geiderman JM, Schears RM, Bookman KJ. Emergency department crowding, part 1-concept, causes, and moral consequences. Ann Emerg Med. 2009;53(5):605-11. doi:10.1016/j. annemergmed.2008.09.019.

9. Asplin BR, Magid DJ, Rhodes KV, Solberg LI, Lurie N, Camargo Jr CA. A conceptual model of emergency department crowding. Ann Emerg Med. 2003;42(2):173-80. doi:10.1067/mem.2003.302.

10. Fletcher A, Worthington D. What is a 'generic' hospital model?-a comparison of 'generic' and 'specific' hospital models of emergency patient flows. 2009(1386-9620 (Print)).

11. Lewis RJ. Modeling complex systems: gaining valid insights and avoiding mathematical delusions. Acad Emerg Med. 2007;14(9):795-8. doi:10.1197/j. aem.2007.06.008.

12. Hwang U, McCarthy ML, Aronsky D, Asplin B, Crane PW, Craven CK, et al. Measures of crowding in the emergency department: a systematic review. Acad Emerg Med. 2011;18(5):527-38. doi:10.1111/j.1553-2712.2011.01054.x.

13. McCarthy ML, Aronsky D, Jones ID, Miner JR, Band RA, Baren JM, et al. The emergency department occupancy rate: a simple measure of emergency department crowding? Ann Emerg Med. 2008;51(1):15-24. doi:10.1016/j. annemergmed.2007.09.003. e1-2.

14. Pines JM. The left-without-being-seen rate: an imperfect measure of emergency department crowding. Acad Emerg Med. 2006;13(7):807. doi:10. 1197/j.aem.2006.02.004. author reply -8.

15. Weiss SJ, Derlet R, Arndahl J, Ernst AA, Richards J, Fernandez-Frackelton M, et al. Estimating the degree of emergency department overcrowding in academic medical centers: results of the National ED Overcrowding Study (NEDOCS). Acad Emerg Med. 2004;11(1):38-50.

16. Sestoft B, Rohde CV. The complexity of the implementation of an observation and tracking chart for critical illness. Ugeskr, Laeger. 2011(1603-6824 (Electronic)).

17. EDs. Danish Emergency Process Triage. Danish Emergency Departments. http://deptriage.dk/. Accessed 20 Apr 2015.

18. Crane PW, Zhou Y, Sun Y, Lin L, Schneider SM. Entropy: A conceptual approach to measuring situation-level workload within emergency care and its relationship to emergency department crowding. J Emerg Med. 2014. doi:10.1016/j.jemermed.2013.08.113.

19. Bernstein SL, Verghese V, Leung W, Lunney AT, Perez I. Development and validation of a new index to measure emergency department crowding. Acad Emerg Med. 2003;10(9):938-42.

20. Epstein SK, Tian L. Development of an emergency department work score to predict ambulance diversion. Acad Emerg Med. 2006;13(4):421-6. doi:10. 1197/j.aem.2005.11.081.

21. Beniuk K, Boyle AA, Clarkson PJ. Emergency department crowding: prioritising quantified crowding measures using a Delphi study. Emerg Med J. 2012;29(11):868-71. doi:10.1136/emermed-2011-200646.

22. Lin D, Patrick J, Labeau F. Estimating the waiting time of multi-priority emergency patients with downstream blocking. Health Care Manag Sci. 2014;17(1):88-99. doi:10.1007/s10729-013-9241-3.

23. Hurwitz JE, Lee JA, Lopiano KK, McKinley SA, Keesling J, Tyndall JA. A flexible simulation platform to quantify and manage emergency department crowding. BMC Med Inform Decis Mak. 2014;14:50. doi:10. 1186/1472-6947-14-50. 
24. McCarthy ML, Ding R, Pines JM, Zeger SL. Comparison of methods for measuring crowding and its effects on length of stay in the emergency department. Acad Emerg Med. 2011;18(12):1269-77. doi:10.1111/j.1553-2712. 2011.01232.x.

25. Wang $H$, Robinson RD, Bunch $K$, Huggins CA, Watson $K$, Jayswal RD, et al. The inaccuracy of determining overcrowding status by using the National ED overcrowding study tool. Am J Emerg Med. 2014;32(10):1230-6. doi:10. 1016/j.ajem.2014.07.032.

26. Pines JM, Hilton JA, Weber EJ, Alkemade AJ, Al Shabanah H, Anderson PD, et al. International perspectives on emergency department crowding. Acad Emerg Med. 2011;18(12):1358-70. doi:10.1111/j.1553-2712.2011.01235.x.

27. Pines JM, Decker SL, Hu T. Exogenous predictors of national performance measures for emergency department crowding. Ann Emerg Med. 2012; 60(3):293-8. doi:10.1016/j.annemergmed.2012.01.024.

28. Hoot NR, Zhou C, Jones I, Aronsky D. Measuring and forecasting emergency department crowding in real time. Ann Emerg Med. 2007;49(6):747-55. doi: 10.1016/j.annemergmed.2007.01.017.

29. Jones SS, Allen TL, Flottemesch TJ, Welch SJ. An independent evaluation of four quantitative emergency department crowding scales. Acad Emerg Med. 2006;13(11):1204-11. doi:10.1197/j.aem.2006.05.021.

30. Flottemesch TJ, Gordon BD, Jones SS. Advanced statistics: developing a formal model of emergency department census and defining operational efficiency. Acad Emerg Med. 2007;14(9):799-809. doi:10. 1197/j.aem.2007.05.011.

31. Madsen $\mathrm{TL}$, Kofoed-Enevoldsen A. Five easy equations for patient flow through an emergency department. Dan Med Bull. 2011:58(10):A4318.

32. McHugh M, van Dyke K, McClelland M, Moss D. Improving patient flow and reducing emergency department crowding: a guide for hospitals. Rockville: Agency for Healthcare Research and Quality; 2011.

33. Bagust A, Place M, Posnett JW. Dynamics of bed use in accommodating emergency admissions: stochastic simulation model. BMJ. 1999;319(7203):155-8.

34. Sorensen HT, Sabroe S, Olsen J. A framework for evaluation of secondary data sources for epidemiological research. Int J Epidemiol. 1996;25(2):435-42.

35. von Elm E, Altman DG, Egger M, Pocock SJ, Gøtzsche PC, Vandenbroucke JP. The Strengthening the Reporting of Observational Studies in Epidemiology (STROBE) statement: guidelines for reporting observational studies. Lancet. 370(9596):1453-7. http://dx.doi.org/10.1016/S01406736(07)61602-X.

\section{Submit your next manuscript to BioMed Central and we will help you at every step:}

- We accept pre-submission inquiries

- Our selector tool helps you to find the most relevant journal

- We provide round the clock customer support

- Convenient online submission

- Thorough peer review

- Inclusion in PubMed and all major indexing services

- Maximum visibility for your research

Submit your manuscript at www.biomedcentral.com/submit
Biomed Central 